\title{
Espacios objetivos y subjetivos de la movilidad cotidiana urbana
}

\author{
Jorge Espinoza Nanjarí*
}

\begin{abstract}
There are the need to generate cartographic models faced to the visualization of the daily mobility of the population of Santiago de Chile, which is framed inside the subjective and personal landscape of the above mentioned mobility defines and determines. It is possible to demonstrate a close link with urban problems that they tend to transform in endemic, such as the transport congestion, the demographic concentration and consequently, the decrease of the mobility.

To achieve this objective, it is necessary to have information originated from direct sources linked to the cognitive, perceptive and appraisal processes that are deeply rooted in the context of the subjectivity and routine character in which the urban inhabitants take its decisions of spatial behavior.
\end{abstract}

Key words: daily mobility, subjective spaces, urban problems.

\section{Resumen}

Se plantea la necesidad de generar modelos cartográficos orientados a la visualización de la movilidad cotidiana de la población de Santiago de Chile, que se enmarca dentro del paisaje subjetivo y personal que dicha movilidad define y condiciona, donde se puede evidenciar una estrecha vinculación con problemas urbanos que tienden a transformarse en endémicos, como lo son la congestión vehicular, la concentración demográfica y por consiguiente, la disminución de la movilidad.

Para lograr este objetivo, es necesario poder contar con información proveniente de fuentes directas vinculadas íntimamente a los procesos cognitivos, perceptivos y valorativos que están enraizados en el ambiente de la subjetividad y cotidianeidad en que los habitantes urbanos toman sus decisiones de comportamiento espacial.

Palabras claves: movilidad cotidiana, espacios subjetivos, problemas urbanos.

* Doctor, Departamento de Cartografía, Universidad Tecnológica Metropolitana de Chile, correo electrónico: jespinoz@utem.cl 


\section{Introducción}

Los dinámicos y continuos procesos de transformación interior que han caracterizado a las ciudades latinoamericanas en los últimos periodos históricos de su evolución, evidencian en la actualidad un paisaje urbano que se ha vuelto considerablemente complejo, en el que se yuxtaponen distintas áreas y dimensiones con funcionalidad y dinámica diferente, conformando un puzzle urbano de marcada heterogeneidad que se hace cada vez menos legible.

Además, se debe agregar un enorme crecimiento demográfico, el que produce inevitablemente una constante expansión física de las ciudades, las que continúan sometidas a importantes tensiones de acondicionamiento interior de extensión periférica, dentro de las cuales destacan, sin duda, los problemas de movilidad, los que se han agudizado en los últimos años, en especial, como resultado de un crecimiento urbano desorganizado y de una expansión rápida e inusitada en el uso del automóvil privado, junto a un alto grado de desorganización del transporte público, traducidos en significativos impactos ambientales que afectan negativamente la vida urbana cotidiana y a la calidad de vida de su población.

La magnitud de la cantidad de viajes que se realizan diariamente en la ciudad de Santiago sobre una insuficiente estructura vial, desde el punto de vista cuantitativo y cualitativo, impacta inevitablemente y en algunos casos en forma dramática a extensas áreas urbanas, donde la población se ve considerablemente afectada en su movilidad habitual y por ende, en sus actividades cotidianas.

Así, durante los últimos años se ha evidenciado un deterioro progresivo de sus condiciones de circulación, expresadas principalmente en la congestión vial. Los tiempos de viaje han experimentado un rápido crecimiento y las vías están alcanzando altos grados de ocupación y saturación, hasta ahora inéditos.

De esta manera, la distancia que separa a los lugares donde se realizan las diferentes actividades económicas y sociales se ha mantenido en crecimiento en los últimos decenios, como consecuencia de los avances tecnológicos y organizacionales.

Asimismo, las razones que motivan los desplazamientos se han incrementado, pasando del "obligado commuting" (Mondrís, 1992) al cotidiano comprar, pago de servicios, trasladar a niños a sus colegios o acceder a bienes culturales y sociales que requieren el uso de medios de transporte motorizado.

Los viajes diarios implican sacrificios en tiempo de descanso, de consumo o de trabajo remunerado, aspecto social que afecta con mayor severidad a los más pobres, cuyos traslados se efectúan en condiciones más inconfortables, con mayores tiempos de desplazamiento el que incluye además con frecuencia realizar transbordos.

Históricamente la periferia urbana de la ciudad de Santiago tendía a ser monofuncional, particularmente residencial, con un alto nivel de segregación social, la 
que ha derivado en su desarrollo a una nueva periferia continua que es multifuncional, la que presenta variados usos del suelo urbano y en algunos casos, sectores sociales diversos y heterogéneos, constituyendo así una nueva experiencia en la consolidación del espacio suburbano santiaguino.

Ejemplos de lo anterior son las nuevas suburbanizaciones residenciales que cuentan con centros comerciales, gimnasios, sedes bancarias, centros médicos y otros servicios urbanos localizados tradicionalmente en las áreas centrales de la ciudad.

Lo anterior, plantea la necesidad de conocer y analizar el patrón de movilidad que ha generado la transición del modelo monocéntrico al policéntrico, con la emergencia de nuevas áreas centrales expandidas por la ciudad. Estas nuevas periferias emergidas en los últimos años, han sido el resultado de importantes movimientos poblacionales intraurbanos, acompañados de la localización de diversos centros comerciales excéntricos en los cuatro puntos cardinales de Santiago, los que han alterado los flujos y la dependencia histórica con el centro comercial tradicional, para una diversidad de viajes derivados de subjetivas, personales y cotidianas motivaciones que no tienen relación necesariamente con las actividades de trabajo o de estudio.

Por otra parte, es importante considerar, que los sistemas públicos de transporte fueron concebidos y diseñados cuando el elemento dominante de la ciudad era el centro, que concentraba las principales áreas del comercio y del trabajo.

La gran mayoría de los Malls, de las áreas comerciales e industriales, de los supermercados, de los establecimientos de salud y educación han sido construidos fuera del centro de la ciudad, estructuras que dependen fuertemente de la existencia de los automóviles. El resultado de este fenómeno, es que se han incrementado significativamente las distancias por recorrer y se ha reducido en forma considerable la importancia de los viajes al centro de la ciudad (Kreime, 2006).

Así, el resultado ha sido que "gran cantidad de personas está lejos de todo" y "las ciudades se han transformado para servir a los automóviles y no a las personas", imponiéndose éstos como un factor determinante en la nueva configuración de los espacios urbanos. Las personas viven lejos de su trabajo, de su lugar de estudio, del supermercado, de los centros comerciales y de servicios.

En los últimos años y en particular desde el inicio de la década de los noventa, el aumento de la demanda de transporte y del tránsito vial ha provocado mayor congestión, demoras excesivas, accidentes y diversos problemas ambientales.

La congestión de tránsito se ha transformado en un severo flagelo, que está presente en la mayoría de los países industrializados como también en aquellos en desarrollo, afectando tanto a automovilistas como a usuarios del transporte colectivo, provocando una serie de efectos para la sociedad y una amenaza en aumento para la calidad de vida urbana. 


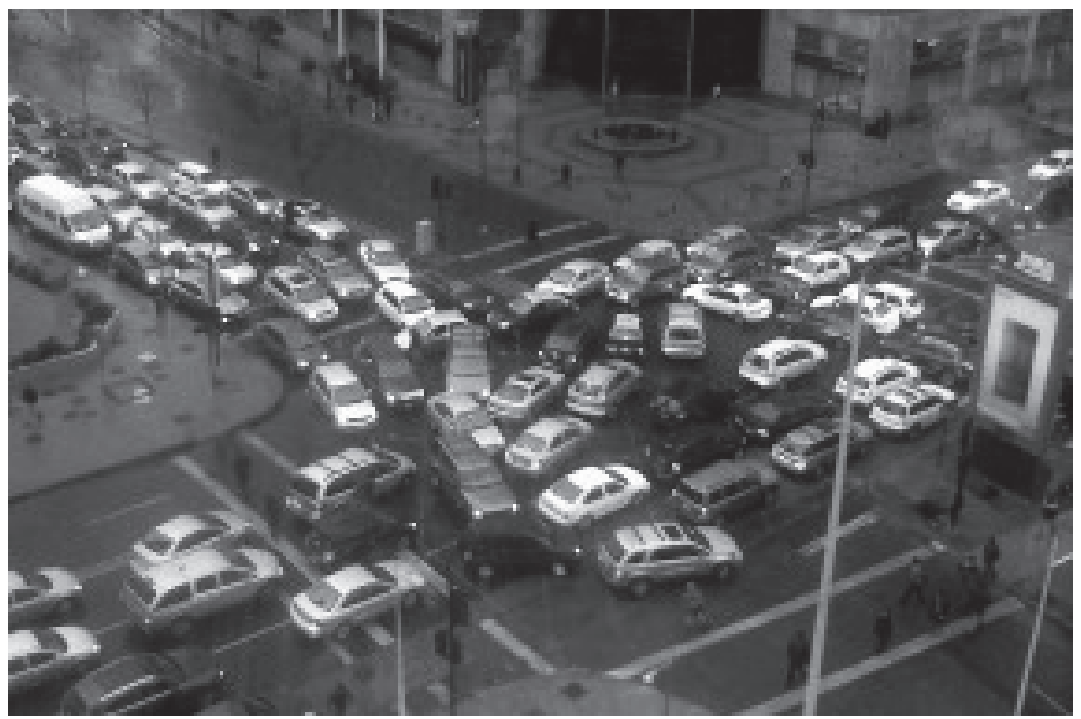

Figura 1. Congestión vehicular y colapso de vías.

Esta situación, puede ser explicada debido a que las últimas décadas han visto un aumento vertiginoso de la cantidad de vehículos motorizados, especialmente en los países en vías de desarrollo, derivado de diversos factores, como el aumento del poder adquisitivo de las clases socioeconómicas de ingresos medios, el mayor acceso al crédito, la reducción relativa de los precios de venta y una oferta de vehículos usados.

Este creciente acceso al automóvil, ha posibilitado una mayor movilidad espacial individual lo que, junto al crecimiento de la población urbana, la menor cantidad de habitantes por hogar, a la escasa aplicación de políticas de transporte urbano y probablemente "debido a la imagen crecientemente atractiva en el mundo en desarrollo hacia un estilo de vida que tiene al automóvil por elemento esencial" (Gakenheinemer, 1998), ha potenciado de manera significativa la congestión.

Ya ha sido comprobado que la organización urbana orientada a facilitar el flujo de automóviles conduce indefectiblemente a un aumento incontrolable del tráfico y de la congestión, estableciéndose así un círculo vicioso en que los vehículos demandan nuevas autopistas y estacionamientos, más espacio urbano que siempre es insuficiente porque, a la vez, promueve el uso de más coches, que finalmente igual colapsan las infraestructuras construidas y además exigen otras nuevas.

Así, la ciudad se ha transformado en función del automóvil, muchos ciudadanos se ven obligados a adaptarse a un ambiente cada vez más difícil y menos suyo. "El medio de transporte se convierte en fin" (Sintes, 2002). 
Una de las consecuencias más evidente de la congestión es aquella relacionada con los tiempos de viaje, especialmente en las horas punta, por otra parte, la lentitud en los desplazamientos exacerba los ánimos y fomenta el comportamiento agresivo de los conductores.

De esta manera, la motorización en la ciudad ha provocado de hecho un grave impacto social, generando un profundo cambio de hábitos y por ende, de comportamiento espacial en las personas, lo que ha contribuido a abrir una importante brecha entre grupos de población en función de la disponibilidad para tener y usar el automóvil.

En términos generales, el mayor crecimiento de la tasa de motorización en comparación con la tasa de crecimiento de la infraestructura, es quizás el factor más relevante que ha contribuido a agravar este multidimensional problema, el que tiene relación con una baja tasa de inversión, tanto de infraestructura vial urbana como interurbana, lo que se traduce en un exceso de demanda por el uso de la infraestructura existente, que es escasa (Romper, 2003).

Es conveniente también mencionar, que la multifuncionalidad ha demandado viajes interperiferia, para los cuales la ciudad aún no está preparada. En estos importantes cambios en el paisaje urbano periférico han tenido gran relevancia los nuevos centros de comercio, servicios y esparcimiento, que Greene y Soler han denominado Subcentros nucleares, que tienen una larga historia de planificación, pero una reciente presencia en la ciudad.

En efecto, el primer Plano Intercomunal de Santiago (PRIS) de 1960 ya indicaba quince subcentros nucleares estratégicamente situados, que proporcionarían equipamiento y servicios urbanos a la periferia santiaguina, con el objeto de disminuir la marcada dependencia de los barrios residenciales con el centro histórico fundacional de la ciudad.

El Plano Regulador Metropolitano de Santiago (PRMS) de 1994, es decir, 34 años después, nuevamente propuso el desarrollo de subcentros nucleares con objetivos y localizaciones muy similares a aquéllos planteados en 1960. El texto señala: "El Plan, en esta materia propone un sistema de once puntos estratégicos denominados Subcentros de Equipamiento Metropolitano, destinados tanto a reunir servicios, como a formar una red de desconcentración de equipamiento, acercándolos a los usuarios" (Ministerio de Vivienda y Urbanismo, MINVU, 1994).

Sin embargo, en la práctica la gran mayoría de los subcentros propuestos por los instrumentos de planificación no se concretaron, o algunos lo hicieron muy débilmente. En su reemplazo se desarrollaron los Malls, que se caracterizan por una dinámica muy diferente, cumplen otras funciones y generan otro tipo de espacio urbano distinto al previsto por los planificadores de los años sesenta.

Malls: cambios en la geografía de oportunidades de la movilidad espacial. 
Las modalidades y magnitud que presenta el crecimiento del espacio urbano de Santiago, de tipo periférico, disperso y fragmentario, constituye una de las características más relevante de los procesos de reorganización urbana actual.

Al respecto, Ortiz y Escolano plantean la hipótesis que en este modelo de crecimiento urbano disperso, la dependencia funcional de las "nuevas periferias" en relación al centro metropolitano es muy acentuada en cuanto a la localización del trabajo y acceso a servicios cualificados. Pero esta relación funcional se evidencia más reducida, y aún inexistente, para productos y servicios cotidianos, debido a la emergencia de centralidades periféricas, cuyos polos son los centros comerciales (Malls).

Es así como, los principales Malls de Santiago se han constituido para el ciudadano común en los nuevos subcentros urbanos, basada esta cualidad principalmente en una nueva concepción de los espacios públicos del habitante, el que ha incrementado su condición de consumidor, transformando las plazas públicas, lugares tradicionales de encuentro y vida social, en una alteración de éstas, constituyéndose en definitiva en un nuevo espacio: el centro comercial o Malls.

En Chile los Malls hicieron su aparición en los años ochenta. En 1981 fue inaugurado Apumanque, centro comercial vecinal y en 1982 el Parque Arauco, centro de carácter regional. Desde esa fecha la construcción de Malls o grandes centros comerciales ha ido en paulatino aumento.

Su éxito popular está relacionado con la función y carácter del centro comercial en general. Además, los Malls se han preocupado de diversificar su oferta, entregando nuevos servicios, como centros médicos y de entretención o institutos de educación superior (Cristoffanini, 2006).

Así, "en los Malls acaece un contacto físico entre las personas. Es posible mirarse, reunirse y dialogar con otros, de manera que el centro comercial se erige como un lugar donde una comunidad de sujetos tiene la capacidad de conocerse. No en vano, los Malls se autodenominan como parques o plazas, lo que denota cómo ellos intentan recuperar del inconsciente colectivo los modos de encuentro inherentes a la modernidad" (Rovira, 2004).

Por otra parte, se localizan en puntos estratégicos de la ciudad y en general, con excelente accesibilidad, en áreas carentes de subcentros urbanos, lo que genera nuevos e importantes flujos de personas desde diferentes sectores de Santiago, lo que ha ejercido una profunda influencia sobre la experiencia del movimiento en la ciudad, al ser el Mall por esencia un concentrador de actividades, lo que se traduce en una buena oportunidad para el usuario de hacer muchas cosas en un solo lugar, evitando así el hecho de tener que desplazarse hacia el centro tradicional para estos efectos (Ponce, 2004). 
De este modo, ha variado significativa y aceleradamente no sólo la cultura de compra de Santiago, sino que también la forma de esparcimiento e interacción, incluyendo en ello los patrones de encuentro y presencia en el espacio urbano.

Dentro de los centros comerciales más importantes de Santiago, es necesario destacar el Mall Plaza Vespucio, inaugurado el 1990 con el objetivo central de impulsar el desarrollo urbano de una comuna con el mayor potencial demográfico en esa fecha, La Florida y constituir el primer Mall del país localizado en una zona de un segmento socioeconómico de clase media (Figura 2).

Corresponde en la actualidad a uno de los centros comerciales más consolidados como subcentro urbano, cuyo radio de influencia alcanza principalmente a diversas comunas del sector sur-oriente y sur-poniente de la ciudad, lo que se traduce en una clara segmentación del mercado, ya que apunta a sectores bastante definidos dentro de la estratificación social, específicamente a sectores emergentes de clase media, con un registro de visitas del $66.5 \%$ del sector socioeconómico medio. El Mall Plaza Vespucio se caracteriza además por contener un espectro programático muy amplio y por su ubicación estratégica.

Debido a la rápida consolidación del Mall Plaza Vespucio como el principal Mall de la zona sur-oriente de la ciudad de Santiago y el más visitado de Chile, la construcción de Malls o grandes centros comerciales ha ido en paulatino aumento, abriendo nuevos mercados de sectores emergentes.

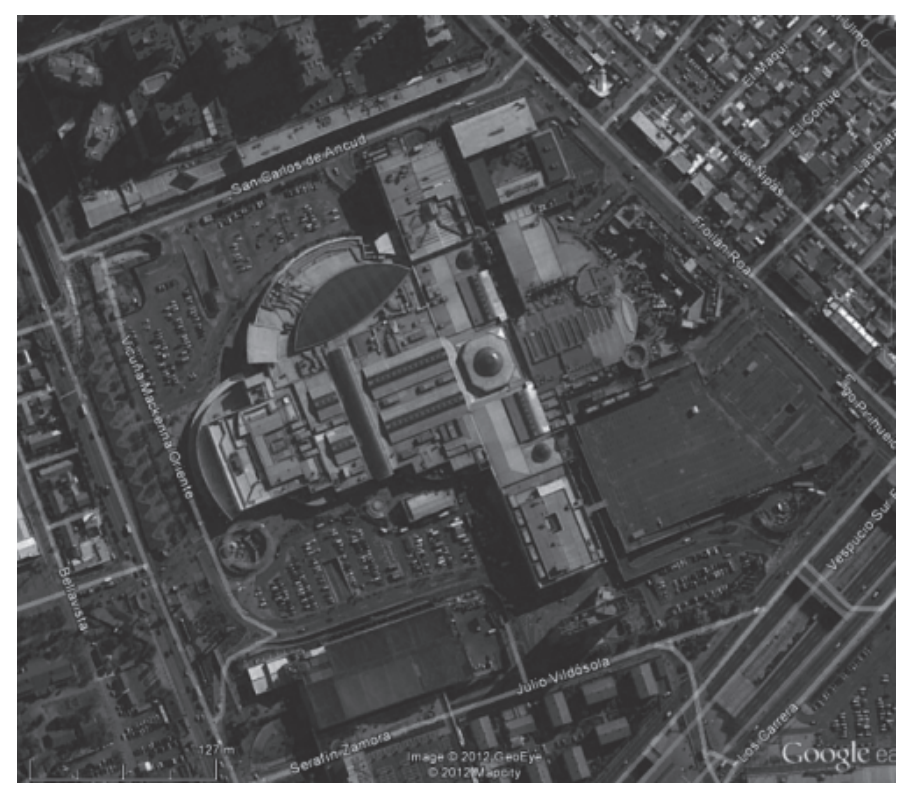

Figura 2. Mall Plaza Vespucio. 
No obstante, lo anterior ha implicado una fuerte presión sobre la red vial y accesibilidad hacia los nuevos centros comerciales, especialmente los Malls, como también hacia el sistema de transporte público, debido a que esta mayor demanda no ha tenido como respuesta una expansión en la oferta que sea capaz de adecuarla, lo que ha traído como resultado una congestión vehicular en permanente aumento.

En efecto, esta diversificación y dinámica que ha experimentado la movilidad de los habitantes de Santiago, ha generado un significativo crecimiento en la demanda por infraestructura vial, la que ha superado con creces la expansión de la oferta de vías. De esta manera, el aumento del número de viajes y el incremento e intensidad en el uso del automóvil, ha derivado en un importante crecimiento en la demanda por infraestructura vial en Santiago.

\section{Búsqueda de racionalidad en la movilidad urbana}

La búsqueda de soluciones efectivas para el problema de la movilidad, es considerada hoy un eje central para la construcción de ciudades habitables. El incontrolable incremento del tráfico, especialmente del automóvil privado, tiene una dramática incidencia en la vida de los ciudadanos: robando espacio público, contaminando el aire, creando ambientes ruidosos, ingratos e inseguros y condicionando los hábitos personales y colectivos.

Por lo tanto, esta problemática para el logro de avances efectivos y permanentes en el tiempo, requiere de una mirada reflexiva, crítica e innovadora, teniéndose en consideración que si el tráfico vehicular es la causa de múltiples problemas, también es consecuencia de muchos factores diferentes, entre los cuales también están aquellos subjetivos, lo que conlleva a la necesidad de intervenir en diversos frentes al mismo tiempo.

El intento de disminuir las necesidades de desplazamientos, se ve claramente entorpecida por el tipo de ciudad que se ha ido imponiendo: "desparramada dividida en sectores monofuncionales, obliga a un enorme aumento de los desplazamientos, imposible de cubrir desde el transporte colectivo. Cuanto más extensa y segregada es la ciudad, más esfuerzo dedicamos al mero trabajo de movernos, de llegar a los sitios" (Sintes, 2002).

Para intentar dar solución a los problemas de movilidad, si bien no se puede "hacer retroceder" a la ciudad desde su condición actual, sí es posible modificar las estrategias y adoptar en la toma de decisiones el criterio de "acercar los servicios y corregir la tendencia a la dispersión espacial, en vez de multiplicar las facilidades de locomoción", en definitiva: favorecer la accesibilidad, en vez de promover la movilidad, adoptándose además formas de desplazamientos compatibles con el ambiente y con todas las personas.

Los esfuerzos deben, por lo tanto, orientarse en este sentido y un buen ejemplo de ello es la experiencia propiciada por el ayuntamiento de Barcelona, el que en 
1998 impulsó el llamado Pacto por la Movilidad, que corresponde a un foco integrado por más de 50 entidades y asociaciones sociales y ciudadanas, que debaten con el objeto de mejorar la movilidad de la ciudad con criterios de sostenibilidad, en base a cifras y objetivos, además de un observatorio que realiza un permanente seguimiento del transporte y de sus efectos (Sintes, 2002).

La preocupación actual es de qué manera se debe enfrentar la inevitable necesidad de seguir desplazándose por la ciudad.

Ya ha sido comprobado que la organización urbana orientada a facilitar el flujo de automóviles conduce indefectiblemente a un aumento incontrolable del tráfico y de la congestión, estableciéndose así un círculo vicioso en que los vehículos demandan nuevas autopistas y estacionamientos, más espacio urbano que siempre es insuficiente porque, a la vez, promueve el uso de más coches, que finalmente igual colapsan las infraestructuras construidas y además exigen otras nuevas.

Los ejes de una nueva movilidad, más racional, que incluya a toda la ciudadanía y que respete el ambiente urbano, junto a la consideración de una oferta de transportes colectivos completa, eficaz y ambientalmente respetuosa, hace necesaria la búsqueda de iniciativas más audaces por parte de las administraciones y un proceso abierto a la participación ciudadana.

Para lograr lo anteriormente indicado, es necesario poder contar con información fiable, que permita una visión cualitativa y cuantitativa más profunda y real de la movilidad urbana, desde fuentes no sólo oficiales provenientes de los organismos encargados tradicionalmente de la planificación y gestión urbana, sino que con especial énfasis, de fuentes directas vinculadas íntimamente a los procesos cognitivos, perceptivos y valorativos que están enraizados en el ambiente de la subjetividad y cotidianeidad en que los habitantes urbanos toman sus decisiones de comportamiento espacial y que, en su conjunto, se traducen finalmente en los desplazamientos, racionales o no racionales, que con su particular dinámica y compleja estructura constituyen el eje central de la problemática de la congestión, tanto demográfica como vehicular.

Así es como, "la vida urbana como un devenir está en movimiento, en el hecho continuo de desplazarse. Por eso el espacio se constituye en escenario del comportamiento cotidiano" (De Castro, 1997). Este comportamiento está regido por desplazamientos que constituyen una fuente de información permanente para reconstruir y actualizar la imagen del entorno urbano.

\section{Movilidad urbana}

Como los desplazamientos en la realidad implican previamente desplazamientos mentales, se debe considerar la movilidad espacial de la población con relación a todas aquellas actividades que motivan voluntaria o forzadamente un desplazamiento, ya sea dentro de las áreas aledañas a su lugar de residencia o hacia, coincidentes 
o diferenciados, puntos y sectores que presentan distintas funcionalidades, localizados en diferentes partes de la ciudad.

En consecuencia, los hábitos de movilidad que serán abordados metodológicamente en el proyecto CART 022013 "Cartografía de la movilidad cotidiana, espacios subjetivos y problemas urbanos", a través de la visualización cartográfica y análisis espacial, han sido determinados en base a tres dimensiones:

- Flujos de desplazamientos habituales en base a las principales vías de circulación de los habitantes, según el lugar de residencia, las características sociodemográficas y la dinámica temporal que caracteriza a su movilidad a través de la ciudad teniendo como lugar de destino y motivo de estudio específico, el Mall Plaza Vespucio que, como ya fue señalado, por su importancia y cobertura a nivel de ciudad, por la oferta de un espectro programático muy amplio y por su ubicación estratégica, se ha estimado de interés seleccionarlo como área de estudio para la investigación.

Es conveniente señalar, que en esta movilidad, junto a la motivación expresada en la valoración que las personas hacen de este centro comercial, la estructura de la red vial juega un papel fundamental como elemento incentivador e indefectible en los desplazamientos que efectúa la población dentro de su espacio vivencial.

De esta manera, la red vial se constituye en un verdadero "mapa operacional", en base al cual el habitante aprehende y valora su espacio, toma decisiones de acción concretas y por último realiza sus diversas actividades habituales, las que se encuentran condicionadas por la estructura y funcionalidad que presenta esta red, como también por la existencia y cantidad en dichas vías de servicio de locomoción colectiva, que representa el principal medio de transporte para las personas que se desplazan por la ciudad.

- Medios de transporte que utilizan las personas en sus desplazamientos habituales, factor que orienta y condiciona la autonomía en las decisiones de su movilidad dentro del área urbana. Se establecen claramente las diferencias cualitativas y cuantitativas de los diferentes flujos, a través de las vías jerarquizadas de acuerdo al nivel e intensidad del uso que de ellas hace la población en sus desplazamientos cotidianos.

- Lugar de destino: Mall Plaza Vespucio. Considerando la frecuencia de menciones vinculadas a la satisfacción de diferentes necesidades básicas y específicas de los residentes en distintos sectores de la ciudad, que han sido establecidos como "zonas de origen" y que han manifestado su preferencia por este centro comercial a través una amplia variedad de razones, que son el producto de la valoración subjetiva y personal que las personas hacen de la oferta de infraestructura y servicios existentes en él y que permiten establecer los componentes diferenciadores y comunes de las decisiones espaciales. 
La visualización cartográfica del significativo flujo de población que se desplaza hacia este centro comercial atributado con una alta preferencia, como asimismo las diversas razones que motivan estos desplazamientos, deberá dejar de manifiesto la multifuncionalidad que éste cumple para satisfacer diversas necesidades de un porcentaje importante de los habitantes de la ciudad, lo que se deriva del grado de valoración y del uso efectivo que ellos hacen de dicho centro, dentro de los límites que establece la percepción subjetiva que éstos tienen del espacio urbano, es decir de su espacio vivencial o cotidiano.

Por otra parte, es necesario también tener presente que este espacio cotidiano es "un espacio egocéntrico, cerrado en función de la experiencia personal y vital del hombre", como asimismo incorpora a la vez un "sentimiento de pertenencia" y la valoración del espacio como resultado de la asignación subjetiva y diferenciada de valores del mismo (Valenti, 1983).

Esta fracción del territorio de una ciudad, con cierta fisonomía propia, que constituye el espacio cotidiano de una determinada comunidad, puede ser dado a una división administrativa como la comuna, pero muchas de las veces es independiente al abstracto límite administrativo.

Es por esta razón, que se estima necesario poder configurar, conocer y comprender la trascendencia que tienen estos espacios subjetivos del entorno familiar, dentro de los cuales las personas intentan satisfacer sus necesidades cotidianas y que derivada de la incapacidad de respuesta que este entorno pueda evidenciar para satisfacerlas, las personas toman las decisiones de acción sobre el resto del espacio urbano, en un proceso de aprendizaje exploratorio, muchas veces espontáneo y de poca coherencia espacial. Proceso que está evidentemente condicionado por la extensión, orientación, calidad y fidelidad de la geoinformación que les proporcionan las imágenes cognitivas que poseen de la ciudad.

La determinación del procedimiento metodológico de la investigación en esta perspectiva, hizo necesario previamente la búsqueda de aplicaciones en diferentes estudios de distintos métodos y técnicas destinadas a recoger, configurar y evaluar información relativa a mapas cognitivos, mapas mentales y comportamiento espacial en áreas urbanas y en particular, se optó por aquéllas propuestas en la tesis doctoral "La diversidad de paisajes subjetivos de Santiago-Chile", entre las cuales el método de Luz y Sombras responde, a nuestro juicio, de manera más efectiva para configurar la percepción subjetiva del espacio urbano cotidiano.

Además, este método contribuye a la necesidad de explorar y analizar nuevas modalidades metodológicas, que se vinculen eficientemente a la visualización cartográfica de los mapas cognitivos y que contribuyan así a una mayor diversificación y profundización en la comprensión y el análisis del complejo proceso de la percepción y del comportamiento espacial que caracteriza a la población que reside en la ciudad de Santiago de Chile. 


\section{Cartografía de luz y sombras}

Corresponde a una visualización cartográfica conformada en base a la identificación o reconocimiento de nodos, sendas e hitos urbanos relevantes, ya sea desde el punto de vista físico, arquitectónico, de patrimonio cultural e histórico, como también funcional, que son el resultado de la lectura semiótica que el habitante urbano hace de su entorno y que se constituyen en la fuente de información básica de orientación y de referencia espacial, que en definitiva conforman y dinamizan su mapa cognitivo.

La primera etapa para la construcción de estas cartas subjetivas, corresponde por consiguiente a la identificación de hitos urbanos significativos para la población, tanto en los lugares periféricos aledaños a los límites comunales que conforman el área de cobertura del Mall, como asimismo en lugares significativos localizados en el interior de dichos sectores, como hitos urbanos de alto valor semiótico asociados a la movilidad cotidiana.

Los niveles de identificación de estos hitos realizada por la población consultada fue visualizada, en una primera instancia, por símbolos puntuales graduados de acuerdo al porcentaje de reconocimiento de cada uno de ellos. Posteriormente, la información gráfica puntual obtenida, fue posible ampliarla a través de la aplicación de dos técnicas de visualización: triangulación e isoperceptas (Figuras 3 y 4$)$.

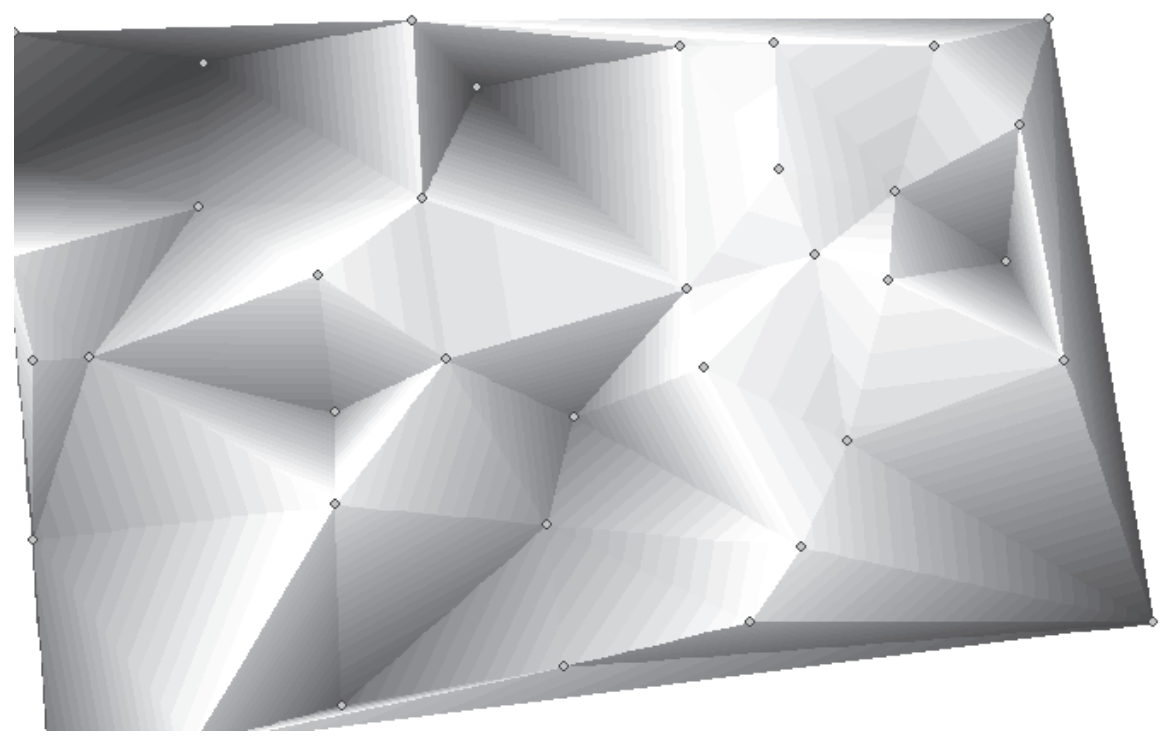

Figura 3. Técnica de interpolación. 


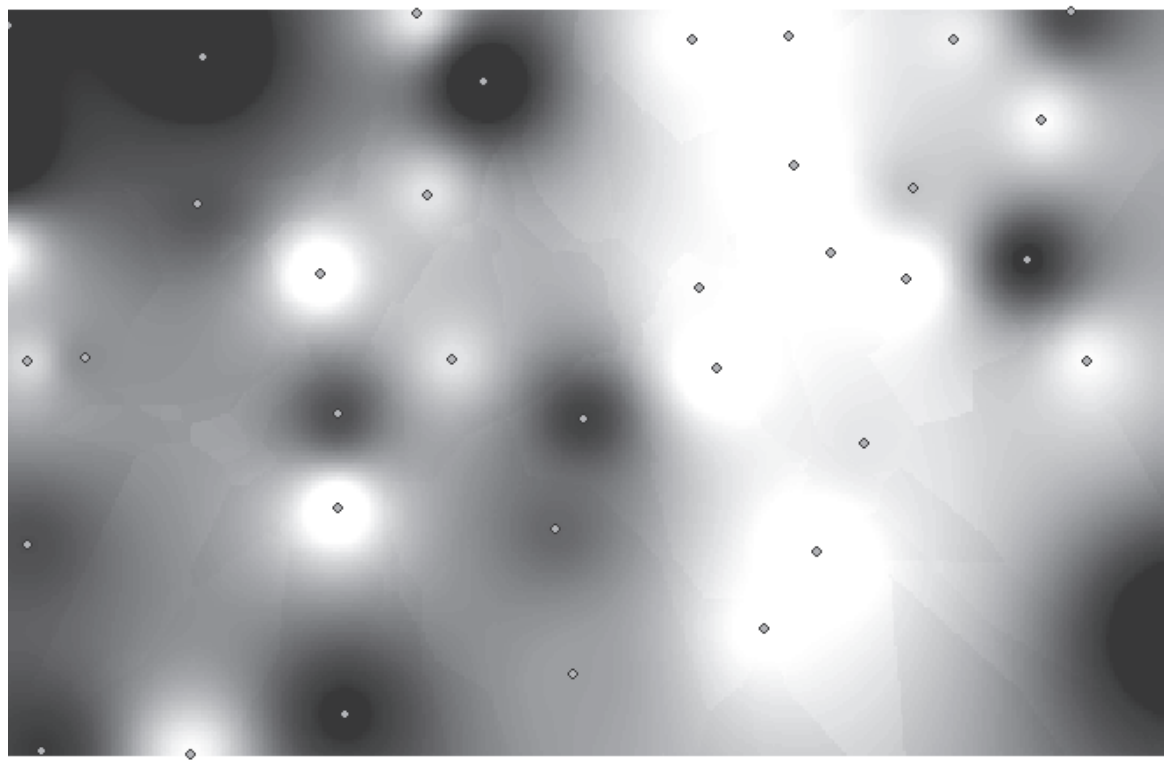

Figura 4. Técnica de isoperceptas.

Finalmente, se contempla una fase de integración y correlación espacial, la que intenta establecer las relaciones existentes en la simbiosis que se presenta entre la visión objetiva de los problemas urbanos en estudio, la que en forma tradicional se considera preferentemente en la planificación urbana en Chile, y la dimensión subjetiva, personal y cotidiana, estrategia de planificación aún no debidamente considerada, que permita comprender y explicar el comportamiento espacial de los habitantes de la ciudad que, en forma consciente o no, son actores activos en la génesis de dichos problemas.

Así, los modelos cartográficos considerados deberán posibilitar una mayor capacidad de comprensión y explicación, en un análisis integrado de los diferentes factores presentes en las dos dimensiones consideradas en el estudio y del grado de racionalidad vinculado éste al proceso de percepción, que se deriva del comportamiento espacial de la población, el que, en definitiva, tiene sus efectos en la creación o modificación de una realidad física y objetiva en permanente transformación y en la configuración de nuevos marcos de referencias espaciales, en cuyo ámbito sea posible reorientar y reorganizar las actividades y acciones, a través de una planificación más integral y por ende, más efectiva. 


\section{Conclusiones}

El interés de esta investigación, que se encuentra en el tercer año de ejecución, se centra fundamentalmente en la visualización cartográfica de aquellos desplazamientos de los habitantes urbanos, que han sido motivados por la multiplicación de los diversos centros comerciales, de servicios, de salud, de recreación y de encuentro social, dentro de los cuales se ha seleccionado el Mall Plaza Vespucio como área de estudio, que son el resultado de este dinámico proceso de transformación que afecta en la actualidad a la ciudad y que motiva una diversidad de viajes derivados de subjetivas y cotidianas razones, las que fueron definidas como "factores independientes" y que no tienen relación con las actividades de trabajo o estudio, las que se presentan más estables en el tiempo y complejas de modificar en el quehacer cotidiano de la población, denominadas "factores dependientes".

Estos desplazamientos habituales que realiza la población de Santiago por motivaciones de carácter independiente, están indefectiblemente condicionados por la estructura y funcionalidad que presenta la red vial de la ciudad, por el lugar geográfico donde reside, por sus características socio-demográficas, por los medios de transportes que utiliza y por la dinámica temporal que evidencia su movilidad conjunta a través del paisaje urbano de la capital.

El análisis preliminar de la visualización cartográfica del comportamiento espacial que presentan las personas en relación al Mall Plaza Vespucio, como lugar de destino, confirma la alta valoración que ellas hacen de la funcionalidad que presenta dicho centro comercial como nueva plaza urbana, donde se entrelazan la cultura, la entretención y el encuentro social, complementados con una moderna infraestructura que proporciona una particular motivación en la población para acceder a los atractivos que ofrecen este espacio público.

Por otra parte, el mapa cognitivo de los residentes en Santiago organiza su estructura a partir de diferentes "espacios de actividad", los que tienen como punto de partida aquellos espacios del entorno familiar dentro de los cuales, en una primera instancia, las personas intentan satisfacer sus necesidades cotidianas y que derivado de la incapacidad de respuesta que este entorno pueda evidenciar para satisfacerlas o de las limitaciones que el mapa cognitivo de los residentes pueda presentar en la coherencia de su estructura y extensión espacial (zonas de luz y sombras), las personas toman las decisiones de acción sobre el resto del espacio urbano, en un proceso de aprendizaje semiológico exploratorio, muchas veces espontáneo y no racional espacialmente. Proceso que evidentemente está condicionado por la calidad, fidelidad y coherencia de la geoinformación que les proporcionan las imágenes mentales que poseen de la ciudad.

Al respecto, se puede concluir que de las vías que presentan en la actualidad un mayor número de puntos críticos de alta congestión, contribuyen a ellas de manera significativa los desplazamientos que realizan los habitantes de la ciudad desde sus 
residencias hasta los lugares de destinos que tienen como motivación la satisfacción de las necesidades denominadas en este estudio como "factores independientes", los que representan, según datos proporcionados por la Secretaría de Planificación de Transporte (SECTRA), el 55.7\% del total de viajes diarios motorizados en Santiago.

El planteamiento teórico-conceptual y el desarrollo metodológico aplicado en la presente investigación, como asimismo el análisis integrado y reflexivo que se intenta realizar en la última etapa del proyecto, permitirán reafirmar la consideración que una parte importante de los problemas urbanos que afectan a la ciudad de Santiago de Chile tienen dos dimensiones, una objetiva, tradicional y pública y otra subjetiva, personal y cotidiana, las que deben ser necesariamente abordadas con la misma profundidad.

La primera dimensión corresponde al espacio métrico u objetivo, donde se centran preferentemente en la actualidad y en algunos casos, en forma exclusiva, las miradas, las acciones y los recursos disponibles.

La segunda dimensión, que ha sido el motivo central de la investigación es el espacio paramétrico, aún no debidamente considerado por los organismos de planificación en el país, en el que coexisten los distintos espacios subjetivos dentro de los cuales subyacen gran parte de las razones que condicionan y permiten explicar el comportamiento espacial de la población urbana y los argumentos que posibilitan establecer su correlación con las problemáticas que se derivan de esta movilidad.

\section{Bibliografía}

Cristoffanini, P. (2006). "La cultura del consumo en América Latina", Sociedad y Discurso, núm. 10.

De Castro, C. (1999). "Mapas cognitivos. Qué son y cómo explorarlos", Scripta Nova, Revista Electrónica de Geografia y Ciencias Sociales, núm. 33, Universidad de Barcelona, España, <www.ub.es/geocrit/sn-33.htm>.

Espinoza, J. et al. (2004). "Cartografía de los espacios subjetivos: una alternativa metodológica para la comprensión del comportamiento espacial de la población urbana”, Instituto Panamericano de Geografía e Historia (IPGH), Informe final, Departamento de Cartografía, Universidad Tecnológica Metropolitana de Chile.

Espinoza, J. (2011). "La diversidad de paisajes subjetivos de Santiago-Chile", tesis doctoral, Universidad de Barcelona.

Gakenheimer, R. (1998). "Los problemas de la movilidad en el mundo en desarro1lo", EURE (Santiago), septiembre, vol. 24, núm. 72.

Greene, M. y Soler, F. (2004). "Santiago: de un proceso acelerado de crecimiento a uno de transformaciones", Facultad de Arquitectura, Diseño y Estudios Urbanos, Pontificia Universidad Católica de Chile, <www.Sitiosur.cl/publicaciones/ Ediciones_sur/02santiago.pdf $>$. 
Kreimer, R. (2006). La tiranía del automóvil. Los costos humanos del desarrollo tecnológico, Ediciones Anarres, Colección Ciencias Sociales, Buenos Aires.

Ortiz, J. y Escolano, S. (2005). "La formación de un modelo policéntrico de la actividad comercial en el Gran Santiago", Revista de Geografia Norte Grande, núm. 34, Pontificia Universidad Católica de Chile.

Ponce, C. (2004). Los grandes nudos viales en la ciudad; contrastes entre Mall y las periferias subyacentes, Centro de Estudios Arquitectónicos, Urbanísticos y del Paisaje, Universidad Central de Chile.

Romper, M. (2003). "Congestión vehicular y tarificación vial", Serie Informe Económico, núm. 137, Libertad y Desarrollo, Chile.

Sintes, M. (2002). "Movilidad racional en las ciudades", Centro Nacional de Educación Ambiental, CENEAM, Chile. 